Théologiques

Théologiques

\title{
Le théologien, " intellectuel organique " et chercheur indépendant : quelle loyauté, quelles tensions, quelle liberté ?
}

\section{Denis Müller}

Volume 14, numéro 1-2, automne 2006

Les lieux de la théologie aujourd’hui

URI : https://id.erudit.org/iderudit/014310ar

DOI : https://doi.org/10.7202/014310ar

Aller au sommaire du numéro

Éditeur(s)

Faculté de théologie et de sciences des religions, Université de Montréal

ISSN

1188-7109 (imprimé)

1492-1413 (numérique)

Découvrir la revue

Citer cet article

Müller, D. (2006). Le théologien, « intellectuel organique » et chercheur indépendant : quelle loyauté, quelles tensions, quelle liberté ? Théologiques, 14(1-2), 61-74. https://doi.org/10.7202/014310ar
Résumé de l'article

L'auteur s'interroge sur la catégorie d'intellectuel organique, que le philosophe marxiste Antonio Gramsci avait appliquée à l'Église catholique. Une relecture de ce concept conduit à développer un portrait critique du théologien comme penseur ecclésial, universitaire et public (à la suite de David Tracy). Cette triple appartenance du théologien, bien comprise, n'est pas contraire à sa liberté de parole et d'action, liberté qui découle de l'Évangile et qui peut s'affirmer dans le contexte d'une modernité revisitée. La tradition protestante offre une vision dynamique et réaliste de cette liberté du théologien, comprise comme loyauté critique, sans rien nier des tensions et des contraintes qui sont les siennes.
Tous droits réservés (C Faculté de théologie et de sciences des religions, Université de Montréal, 2006
Ce document est protégé par la loi sur le droit d'auteur. L'utilisation des services d'Érudit (y compris la reproduction) est assujettie à sa politique d'utilisation que vous pouvez consulter en ligne.

https://apropos.erudit.org/fr/usagers/politique-dutilisation/ 


\section{Le théologien, "intellectuel organique » et chercheur indépendant: quelle loyauté, quelles tensions, quelle liberté ?}

Denis MÜLLER

Faculté de théologie et de sciences des religions

Université de Lausanne ${ }^{1}$

Dans cet exposé, nous nous proposons de méditer sur le statut du théologien chrétien en régime confessionnel et œuménique postmoderne. Un détour historique, mais aussi politico-philosophique, par la notion d'intellectuel organique analysée par le philosophe marxiste critique Antonio Gramsci (1891-1937), nous conduira à nous interroger sur la liberté du théologien, dans la loyauté critique qu'il ou elle entretient avec l'Église, la tradition et la foi. Cela nous amènera à reprendre la question des rapports entre le théologien ecclésial, le théologien académique et le théologien public, selon la distinction proposée en 1981 par David Tracy.

1. «L'intellectuel organique »: une notion pertinente pour approcher le statut du théologien?

La notion d'intellectuel organique a été introduite et développée par le célèbre théoricien marxiste Antonio Gramsci. Selon lui, la famille, l'école, l'Église, les partis, les professions, les institutions scientifique, universitaire, artistique, les moyens de communication de masse constituent une forme de domination de classe. Celle-ci se réalise au sein d'un mode de vie et de pensée, d'une forme de la culture et des rapports sociaux. Dans les plis et replis de l'existence quotidienne se fabriquent l'adhésion et le consentement qui assurent l'hégémonie. Pour le théoricien marxiste, le pouvoir de l'État n'est donc pas la source de l'hégémonie, mais son résultat. En cela, Gramsci ne fait (selon lui)

1. L'auteur a été président de l'Association de théologiens pour l'étude de la morale (Paris) de 1999 à 2005. Il s'exprime ici en son nom personnel. 
que suivre l'histoire de l'Église catholique, modèle type de l'institution qui a multiplié au cours des siècles les formes de domination.

Tout groupe social, qui nait sur le terrain originaire d'une fonction essentielle dans le monde de la production économique, se crée, en même temps, de façon organique, une ou plusieurs couches d'intellectuels qui lui apportent homogénéité et conscience de sa propre fonction, non seulement dans le domaine économique, mais également dans le domaine social et politique (Gramsci 1978, 309).

Un des traits caractéristiques les plus importants de chaque groupe qui cherche à atteindre le pouvoir est la lutte qu'il mène pour assimiler et conquérir «idéologiquement » les intellectuels traditionnels, assimilation et conquête qui sont d'autant plus rapides et efficaces que ce groupe donné élabore davantage, en même temps, ses intellectuels organiques. $(1975,604)$

Gramsci explique en quoi la notion d'hégémonie est indispensable à tout processus de conquête du pouvoir. Dans sa pensée, les intellectuels jouent un rôle déterminant dans la constitution et le maintien de cette hégémonie. Schématiquement, le parti communiste serait à l'intellectuel marxiste ce que l'Église est à l'intellectuel dit traditionnel catholique. Gramsci montre déjà la dimension religieuse du parti, bien que le parti aspire à devenir l'État là où l'Église est déjà sinon l'État du moins une instance semblable à l'État. C'est ainsi qu'il théorise la fonction de l'intellectuel organique et la notion de métapolitique. C'est-à-dire que la conquête du pouvoir politique passe d'abord par la conquête de l'hégémonie culturelle.

Dans les sociétés occidentales, il est impossible de prendre le pouvoir politique sans contrôler d'abord le pouvoir culturel. C'est pourquoi il convient d'adapter les théories marxistes de révolution et de dictature du prolétariat à la réalité sociale. De même, la prise du pouvoir étatique à l'aide d'une insurrection politique est impossible dans les mêmes sociétés évoluées, s'il n’y a pas préalablement un long travail idéologique de préparation du terrain au sein de la société civile.

Pour Gramsci, l'avènement du socialisme ne passe ni par le putsch ni par l'affrontement direct, mais par la subversion des esprits. Il faut donc contrôler la culture, parce qu'elle est la clé des valeurs et des idées. En effet, dans nos sociétés, la société civile joue un rôle décisif par rapport au politique, et l'hégémonie idéologique et sociale l'emporte, souvent, sur la domination politique et sur la force.

S'inspirant de Machiavel, Gramsci assigne dès lors une tâche déterminante aux «intellectuels »; par un travail de termite, ils doivent saper les 
valeurs de la société traditionnelle et capitaliste. Ayant une fonction sociale et politique, ils doivent être ralliés ou détruits.

\subsection{En quel sens est-il approprié (sur le plan de la description) et légitime (théologiquement) de dire que le théologien est un intellectuel organique?}

À l'entendre ainsi rappelée, la notion marxiste d'intellectuel organique semble peu adaptée à la condition actuelle des théologiens. Ou alors, elle apparaît tellement critique et subversive qu'elle ne conviendrait qu'à une certaine catégorie de théologiens - le théologien rebelle, critique, dissident. À la limite, seul le théologien protestant, ou crypto-protestant, articulé sur le "principe protestant» (Tillich), autrement dit sur la critique permanente, aurait la possibilité de tirer son épingle du jeu. Sa qualité " organique" se jouerait en somme dans sa capacité à désorganiser l'organe oppressif de la Vérité imposée. La théologie serait subversive et dissidente, de part en part. Ce serait la victoire du principe protestant, contre toute velléité de puissance de la «substance catholique».

L'intellectuel organique, selon Gramsci, est en effet un intellectuel productif au sein du système capitaliste. Il participe d'une culture de la domination.

Gramsci distingue l'intellectuel organique de l'intellectuel traditionnel, dans lequel il range les ecclésiastiques. Le théologien est d'abord un intellectuel traditionnel, au double sens d'indépendant et de rattaché à une tradition (de foi, de pensée). Mais n'est-il pas aussi organique, au sens de rattaché à un pouvoir, à un magistère, à une structure, à un ordre ? C'est cette ambivalence qui donne à penser et dont nous aimerions rendre compte ici de manière différenciée et dialectique, plutôt que sur un mode binaire.

\subsection{S'agirait-il d'une catégorie plus facile à entendre par le théologien protestant que par le théologien catholique?}

On ne peut pas échapper à cette interrogation. Comme théologien protestant, je suis toujours très méfiant quand le protestantisme glisse du côté de la bonne conscience ou de la propre justice. Si le recours analogique et critique à la catégorie gramscienne de l'intellectuel organique avait simplement pour but de conforter le protestantisme dans ses préjugés originaires envers le catholicisme, alors nous aurions succombé nous-même à la tentation que nous dénonçons: nous aurions fondé notre vision de la vérité 
théologale ou éthique sur l'effet fonctionnel produit par l'institution dont nous dépendons. Les Églises protestantes ne sont pas des non-institutions, mais des institutions d'un genre mixte, une sorte de corpus mixtum se tenant à mi-distance de l'ecclesia néotestamentaire rêvée et de l'idéal démocratique moderne. L'institutionnalisation protestante, avec ses caractéristiques de pluralisme et d'éclatement typiques de la modernité, a sécrété des formes sui generis d'intellectuel organique, non seulement sur le plan social, mais aussi dans les types de légitimation théologique auxquels elle a donné naissance.

Deux écueils se sont alors historiquement présentés. D’un côté, le théologien s'est presque entièrement concentré et résorbé dans la seule figure du pasteur, ce qui a eu pour effet paradoxal une intellectualisation de la figure et des pratiques des pasteurs, mais également, de manière quasi simultanée, une désaffection du protestantisme, pasteurs compris, envers l'exigence intellectuelle et théorique de la théologie. Pasteurs et Églises ont déserté la théologie académique, en même temps qu'ils se sont construit une image négative et un repoussoir commode en installant le théologien académique dans une tour d'ivoire souvent plus fantasmée que réellement existante. D'un autre côté, Églises et théologiens, par paresse et par démagogie, ont préféré ne pas distinguer clairement ministres et laïcs et ont fait comme si, la théologie étant le lot de tous sans formation et sans esprit critique, la laïcisation de l'Église était synonyme de suppression du labeur théologique.

Si la difficulté inhérente à cette institutionnalisation protestante diffractée et éclatée a conduit au double écueil que nous venons de signaler, le risque inhérent au catholicisme est tout naturellement d'un tout autre genre.

\subsection{Critique de la conception magistérielle romaine récente du théologien catholique comme intellectuel organique objectif}

Nous pouvons renvoyer ici au motu proprio «Ad Tuendam Fidem » du 18 mai 1998. On peut notamment y lire ceci (les soulignés sont de nous):

A) Le canon 750 du Code de Droit canonique aura désormais deux paragraphes, le premier comprenant le texte du canon actuellement en vigueur, le second comportant un nouveau texte; le texte complet de ce canon 750 sera donc le suivant:

Can. $750, \mathbb{S} 1$. On doit croire de foi divine et catholique tout ce qui est contenu dans la parole de Dieu écrite ou transmise par la tradition, c'est-à- 
dire dans l'unique dépôt de la foi confié à l'Église et qui est en même temps proposé comme divinement révélé par le magistère solennel de l'Église ou par son magistère ordinaire et universel, à savoir ce qui est manifesté par la commune adhésion des fidèles sous la conduite du magistère sacré; tous sont donc tenus d'éviter toute doctrine contraire.

$\mathbb{S} 2$. On doit aussi adopter fermement et faire sien tous les points, et chacun d'eux, de la doctrine concernant la foi ou les moeurs que le magistère de l'Église propose comme définitifs, c'est-à-dire qui sont exigés pour conserver saintement et exposer fidèlement le dépôt de la foi; celui qui repousse ces points qui doivent être tenus pour définitifs s'oppose donc à la doctrine de l'Église catholique.

Dans le canon 1371, n. 1, du Code de Droit canonique, il convient d'ajouter une référence au canon $750, \mathbb{} 2$; le texte de ce canon 1371 sera donc:

Can. 1371. Sera puni d'une juste peine:

$1^{\circ}$ qui, en dehors du cas dont il s'agit au can. $1364, \mathbb{} 1$, enseigne une doctrine condamnée par le Pontife romain ou le Concile œcuménique, ou bien qui rejette avec opiniâtreté un enseignement dont il s'agit au can. $750, \mathbb{} 2$, ou au can. 752, et qui, après avoir reçu une monition du Siège apostolique ou de l'Ordinaire, ne se rétracte pas;

$2^{\circ}$ qui, d'une autre façon, n'obéit pas au Siège apostolique, à l'Ordinaire ou au Supérieur lorsque légitimement il donne un ordre ou porte une défense, et qui, après monition, persiste dans la désobéissance. (Jean-Paul II 1998)

Nous avons là un exemple significatif de la confusion entre les instances théologales de légitimation et les instances ecclésiales de contrôle. On en trouvait déjà le geste dans l'encyclique Veritatis splendor en 1993.

La notion d'intellectuel organique était orientée, chez Gramsci, d'abord vers les institutions. Le Parti ou l'Église exprimaient la quintessence de l'intellectuel organique. Or, nous devons aussi nous interroger sur le fonctionnement du théologien, au sein de l'institution ecclésiastique. Nous découvrons qu'il est à la fois un intellectuel organique objectif et un intellectuel organique subjectif (distinction que nous introduisons librement, sans qu'elle s'appuie sur la lettre de la pensée de Gramsci).

Le théologien n'est pas seulement ici un intellectuel organique au sens subjectif, c'est-à-dire en tant qu'un sujet critique et un acteur libre, adhérant au corps de doctrine régulant l'institution de son choix et participant à sa discussion rationnelle et communautaire, il est aussi contraint de fonctionner comme un intellectuel organique au sens objectif, en ce sens négatif 
et répressif qu'il se voit intimer l'ordre d'obéir sans discussion et de se rétracter en cas de désaccord.

\section{Esquisse de reprise systématique}

\subsection{La problématique de la loyauté critique}

En partant de la notion d'intellectuel organique et en essayant de la mettre au service de la compréhension des rôles et des visées du théologien, nous avons pris le parti et le risque de lier le théologien à l'institution, surtout et avant tout à l'institution-Église. Or, la problématique de la loyauté critique du théologien ne se pose pas de manière binaire dans un vis-à-vis simpliste entre un sujet isolé et une institution à caractère purement objectif. S'il y a conflit potentiel et loyauté en jeu dans la relation du théologien et de l'Église, c'est bien parce que l'Église elle-même est porteuse d'un projet de sens et d'une autocompréhension de type doctrinal et potentiellement idéologique.

Pour le dire de manière lapidaire et heuristique, la loyauté du théologien envers l'institution-Église ne peut être qu'une loyauté critique, pour la bonne et simple raison que le référent de base commun à l'Église et au théologien - l'Évangile, le dépôt de la foi, etc. — se tient en position de Tiers entre le théologien et l'institution. La source de la critique inhérente à la loyauté du théologien ne se tient pas d'abord dans la rationalité critique de la modernité, mais bien dans la charge symbolique critique de l'Évangile et de la foi par rapport à toute transmission historico-traditionnelle et à toute institutionnalisation de leur visée.

\subsection{La question des médiations}

On pourrait baliser la problématique œcuménique des médiations de la manière idéale typique et forcément réductrice et simplificatrice suivante:

- La maladie congénitale du protestantisme revient à un surinvestissement de la subjectivité, avec pour effet une défiance de principe envers les médiations (tradition et institution).

- La maladie congénitale du catholicisme revient à un surinvestissement de la tradition et de l'institution, au détriment de l'affirmation de la spontanéité de la conscience morale et de la liberté critique des croyants et des sujets individuels. 
En termes plus systématiques, on pourrait reformuler la question de la manière suivante:

- Au sujet de la tradition: la tradition, comme flux historique et pluriel de l'interprétation et de la réception de l'Évangile, constitue une médiation inéluctable et indispensable entre le fondement évangélique de la théologie et son actualisation socioculturelle et politique contemporaine. La tradition demeure ainsi une médiation nécessaire, mais non suffisante, de la compréhension critique de la vérité chrétienne.

- Au sujet de l'institution: le fondement de l'institution ecclésiale et de son autorité spécifique est l'Évangile. L'Évangile demeure par conséquent l'instance critique permanente et récurrente de légitimation et de réforme de l'autorité ecclésiale.

- Au sujet du statut du théologien et de la théologienne: les théologiens et les théologiennes ont une dette première envers l'Évangile et une dette dérivée envers l'institution et la tradition, à la construction de laquelle ils contribuent, par rapport à laquelle, toutefois, ils ou elles ne sont jamais liés de manière ultime ou dernière, mais seulement de manière avant-dernière.

- Au sujet de la communauté herméneutique de la théologie: aucun théologien n'a le dernier mot sur la juste interprétation de l'Évangile. La communauté ecclésiale et scientifique des théologiens et des théologiennes constitue pour ainsi dire un élément médiateur entre le sensus fidelium et l'expression œcuménique de la foi commune formulée par l'autorité synodale, conciliaire ou «magistérielle».

\subsection{Liberté théologale et liberté moderne}

- La liberté du théologien moderne se joue dans l'espace mobile de la liberté critique de la rationalité moderne et de la liberté herméneutique des croyants comme individus et comme membres de la communauté ecclésiale.

- Cette liberté interprétative des croyants n'est pas une simple réaction à la situation des Lumières et au contexte moderne et postmoderne de la sortie de la religion, elle émane aussi, à un niveau plus fondamental, de la constitution plurielle et conflictuelle du canon des Écritures et de sa réception traditionnelle et institutionnelle. 


\section{Le théologien ecclésial, le théologien académique et le théologien public}

\subsection{Sens de la distinction}

Je m'inspire ici très librement de la célèbre distinction introduite par David Tracy (1981) et discutée ailleurs (Müller 1999).

Notons que le portrait, par Tracy, du théologien comme orienté vers un triple domaine de réalité et vers un triple public: la société, l'université et l'Église, relevait d'une approche sociologique et fonctionnelle. Ce "portrait social » n'était pas le dernier mot de Tracy. Venait en effet s'y ajouter un «portrait théologique » (1981, 47s.), également scandé selon un rythme ternaire: le théologien, dans sa vision spécifique et interne de la tâche théologique, se décline en théologien fondamental, systématique et pratique. Le théologien fondamental a affaire avec la rationalité; le théologien systématique, avec l'intelligence de la foi; le théologien pratique, avec la pertinence sociale et culturelle de la foi. On le voit aisément: il y a correspondance stricte entre les trois publics du théologien dans sa fonction sociale et les trois visées du théologien dans sa mission théologale:

\begin{tabular}{|l|l|}
\hline Portrait social & Portrait théologique \\
\hline Public: l'université & Théologie fondamentale \\
\hline Public: l'Église & Théologie systématique \\
\hline Public: la société & Théologie pratique (ou éthique) \\
\hline
\end{tabular}

Tous les théologiens, toutes les théologiennes ne cumulent pas nécessairement les trois fonctions.

Il y a des théologiens qui ne sont que des théologiens ecclésiaux. On ne parle pas ici de leur formation, qui peut très bien avoir été académique, mais de leur fonction. Ils ou elles travaillent uniquement dans l'Église et ne dépendent que d'elle. Ils ou elles peuvent avoir une mission purement tournée vers l'intérieur de l'Église, mais on notera déjà, à ce niveau, qu'ils ou elles échappent difficilement à la fonction publique de la théologie. Il est extrêmement rare, en effet, qu'une activité interne à l'Église n'ait pas de dimension publique, ne serait-ce que de manière implicite.

Inversement, le théologien académique peut entretenir des liens à géométrie variable aussi bien avec l'Église qu'avec l'espace public. 


\subsection{Perte de tout lien organique avec l'Église?}

L'idéal type du théologien académique "libéral » ou "libéré ", correspondant au paradigme schématique de l'intellectuel autonome moderne, semble avoir rompu toutes les amarres avec l'organon de l'institution ecclésiastique et être ainsi devenu un intellectuel apparemment "anorganique». Pourtant, son vivier naturel ou son biotope, loin de s'estomper complètement, n'a fait que changer de nature. L'intellectuel organique a beau avoir changé de relation organique, de milieu, il n'en demeure pas moins objectivement et subjectivement corrélé à une instance de contrôle, l'université, ou les pouvoirs publics à qui l'université doit sa légitimation et sa reconnaissance (à la fois matérielle et symbolique).

\subsection{Détachement ou dépendance envers l'Académie ou les pouvoirs publics soutenant les universités?}

Sur l'autre versant, le théologien académique - semblable sur ce point à tout universitaire - peut occuper une posture subjective antagoniste, au sein même de l'université : il peut en effet se comprendre comme témoin de la liberté académique au sens le plus strict et le plus pur, ne devant rien à quiconque et surtout pas à ceux qui le rémunèrent et dont il dépend administrativement. Il serait alors une sorte de chercheur religieux autonome, ressemblant à s'y méprendre à certains de ses collègues de sciences des religions (qu'on supposera ici, pour la simplicité provisoire de la démonstration, eux-mêmes axiologiquement neutres et foncièrement anorganiques, illusion trop commode, on l'a dit)!

\subsection{Le théologien public menacé d'isolement?}

Le théologien public contemporain, livré à sa solitude de chercheur religieux, peut donner l'impression de ne rien devoir à personne, ni aux Églises, ni à l'Académie. Or, son orientation vers le public potentiellement universel que constitue la société at large a changé profondément de statut entre la fin du XVIII siècle et le début du XXI ${ }^{e}$ siècle. Dans le modèle classique de la théologie pratique (héritée de Schleiermacher) ou pastorale, le théologien était encore un intellectuel organique polarisé par une double allégeance: sa dette envers l'Église et sa dette envers la société ne posaient pas de problème de fond et ne risquaient que faiblement d'entrer en conflit ouvert: malgré la forte menace qui pesait déjà, depuis les Lumières, sur 
la religion et sur les religions - ce fut, on s'en souviendra, le point de départ de l'attitude apologétique de Schleiermacher, en 1799, "contre les contempteurs (ou les mépriseurs) de la religion» (voir Schleiermacher 2004). Or, de nos jours, l'union sacrée de la société et de la religion ne peut même plus être pensée comme redevable d'une possible corrélation idéale: avec l'avènement de l'ère de la postchrétienté (H.T. Engelhardt fils) ou de la «sortie de la religion» (M. Gauchet), nous sommes entrés de manière apparemment irréversible dans un paradigme révolutionnaire. Le théologien ne peut plus se prendre à rêver de faire se correspondre dans la réalité sociale et culturelle ce qu'il essaie encore tant bien que mal de concilier dans sa dynamique subjective et théorique personnelle. La corrélation objective du social et du religieux étant devenue inaccessible à large échelle, il ne reste plus au théologien public qu'à se transmuer en interprète privé du sens religieux de l'existence.

Sa dette sociale a cependant le désavantage énorme d'être vague, générale, pseudo-universelle. Il est le théologien de toutes et de tous, ce qui peut le conduire à la démagogie, à l'enflure ou à la désarticulation.

On comprend que, dans ces conditions postchrétiennes et postmodernes d'hypersécularisation objective, le théologien soit tenté de se replier sur la contre-objectivité sécurisante de la communauté ecclésiale et sur la pseudo-objectivité agressive d'une rethéologisation radicale des réalités profanes, en passant par la rethéologisation de la raison, de l'éthique et de la politique.

Nous avons bien des raisons de penser qu'un tel repli, à la fois institutionnel et théorique, est paresseux, frileux et contre-productif. D'où une proposition moins réactive et plus confiante.

\subsection{Proposition}

Nous défendons en conclusion une conception dialectique et critique du rôle du théologien, et ce, à deux niveaux : au niveau de la théorie des fonctions, puis à celui de la théorie des instances de légitimation ou des horizons constitutifs de sens.

Cette conception peut se résumer dans le double schéma suivant:

(1) Touchant la théorie des rôles ou des fonctions, le théologien peut avoir en vue, de manière spécifique ou diversifiée :

- le public ecclésial;

- le public académique;

- le public sociétal. 
Son discours subit des changements, selon qu'il parle au nom de l'Église ou en son nom propre. Mais il ne peut jamais parler seulement au nom de l'Église ou seulement en son nom propre. Il engage toujours une certaine conception de la liberté chrétienne en lien avec la tradition, la raison, la vérité et le monde. D'où la nécessité de compléter la théorie des rôles et des publics par une théorie des instances de légitimation.

(2) Touchant la théorie des instances de légitimation ou des horizons constitutifs de sens, le théologien opère trois types de médiation:

- la mise en perspective de la foi, sous ses formes historiques et culturelles changeantes, et de la tradition, qui se diffracte en traditions plurielles et dynamiques;

- l'exercice d'une raison critique au service de l'humanisation de la société et des sujets qui s'y meuvent;

- la visée d'une universalité concrète, surmontant le particularisme des positions de foi et l'universalisme abstrait des laïcités fermées.

Le théologien n'est jamais seulement un exégète, un interprète, un praxéologue ou un dogmaticien. Il est toujours aussi un systématicien devant rendre compte, sur les plans de la théologie et de l'éthique fondamentales, des liens de la tradition chrétienne avec à la fois la raison critique, le conflit des interprétations et la différenciation croissante du monde socioculturel et politique. Il est ainsi exposé à la vérification théorique et pratique du sens concret des prétentions à l'universalité qui traverse l'idéal chrétien. Ce faisant, il ne peut esquiver la question des relations entre liberté, vérité et pouvoir. La splendeur de la vérité n'est jamais accessible hors d'un débat sur la liberté, la pluralité des interprétations et l'analyse critique des effets de pouvoir également présents dans la structure institutionnelle et dans le fonctionnement réel des Églises.

\section{Références}

Demaison, M., dir. (1995), La liberté du théologien. Hommage à Christian Duquoc, Paris, Cerf (Cogitatio Fidei).

Duquoc, C. (1999), "Je crois en l'Église». Précarité institutionnelle et Règne de Dieu, Paris, Cerf (Théologies).

GefFré, C. (1999), Profession Théologien. Quelle pensée chrétienne pour le XXI ${ }^{e}$ siècle? I entretiens avec Gwendoline Jarczyk, Paris, Albin Michel. 
Gramsci, A. (1975), Gramsci dans le texte. De l'avant aux derniers écrits de prison (1916-1935) / recueil de textes réalisé sous la direction de F. Ricci en collaboration avec J. Bramant; textes traduits par J. Bramant, G. Moget, A. Monjo et F. Ricci, Paris, Éditions sociales.

(1978) [italien 1926-1937], Cahiers de prison, t. III : Cahiers 10-13 / trad. par P. Fulchignoni, G. Granel et N. Negri, avant-propos et notes de Robert Paris, Paris, Gallimard (Bibliothèque de philosophie).

Jean-Paul II (1998), Lettre apostolique en forme de Motu Proprio Ad Tuendam Fidem (18 mai 1998), disponible sur le Web à : <www.vatican. va/holy_father/john_paul_ii/motu_proprio/documents/hf_jp-ii_motu-proprio_30061998_ad-tuendam-fidem_fr.html>.

Müller, D. (1999), L'éthique protestante dans la crise de la modernité. Généalogie, critique, reconstruction, Paris / Genève, Cerf (Passages) / Labor et Fides.

(2002), «La bioéthique au péril de Dieu: pour une critique théologique de la maîtrise éthique sur le vivant ", Revue de Théologie et de Philosophie, 134/4, p. 327-340.

- (2005), Karl Barth, Paris, Cerf (Initiation aux théologiens).

Naud, A. (1987), Le magistère incertain, Montréal, Fides.

Portelli, H. (1974), Gramsci et la question religieuse, Paris, Anthropos.

Roberge, R.-M. (1988), «La fonction magistérielle dans l'Église. À propos d'un ouvrage récent ", Laval théologique et philosophique, 44/3, p. 375 391.

Schleiermacher, F.D.E. (2004) [allemand 1799], De la religion. Discours aux personnes cultivées d'entre ses mépriseurs / trad. par B. Reymond, Paris, Van Dieren (Références théologiques).

TraCy, D. (1981), The Analogical Imagination. Christian Theology and the Culture of Pluralism, Londres, SCM Press.

\section{Résumé}

L'auteur s'interroge sur la catégorie d'intellectuel organique, que le philosophe marxiste Antonio Gramsci avait appliquée à l'Église catholique. Une relecture de ce concept conduit à développer un portrait critique du théologien comme penseur ecclésial, universitaire et public (à la suite de David Tracy). Cette triple appartenance du théologien, bien comprise, n'est pas contraire à sa liberté de parole et d'action, liberté qui découle de l'Évangile 
et qui peut s'affirmer dans le contexte d'une modernité revisitée. La tradition protestante offre une vision dynamique et réaliste de cette liberté du théologien, comprise comme loyauté critique, sans rien nier des tensions et des contraintes qui sont les siennes.

\section{Abstract}

The author examines the category of organic intellectual, applied by the Marxist philosopher Antonio Gramsci to the Catholic Church. A new interpretation of this category allows to develop a critical portrait of the theologian as ecclesial, academic and public thinker (following David Tracy). The well-interpreted dependence of the theologian towards church, university and society is not contradictory to his freedom of speech and action. This freedom is a consequence of the Gospel and has a good impact in the context of a revisited modernity. The protestant tradition offers a dynamic and realistic vision of such a freedom of the theologian, understood as critical loyalty, without denying the tensions and the constraints inherent to his or her situation. 